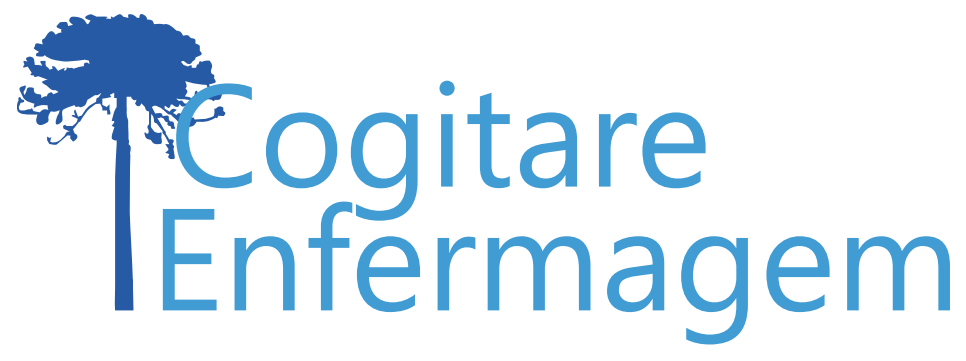

\title{
SAÚDE MENTAL DOS PROFISSIONAIS DE ENFERMAGEM DO BRASIL NO CONTEXTO DA PANDEMIA COVID-19: AÇÃO DO CONSELHO FEDERAL DE ENFERMAGEM
}

Dorisdaia Carvalho de Humerez ${ }^{1}$, Rosali Isabel Barduchi Ohl², Manoel Carlos Neri da Silva ${ }^{3}$

\section{RESUMO}

Objetivo: refletir sobre a saúde mental dos profissionais de enfermagem brasileiros no contexto da pandemia COVID-19.

Desenvolvimento: o atual surto da doença causada pelo Coronavírus, a COVID-19, está ocasionando prejuízos enormes para todo o mundo e tende a provocar pânico generalizado na população. Diante dessa realidade, os profissionais da enfermagem fazem parte de um dos grupos mais afetados, expostos ao risco de contágio e da dor emocional que afeta consideravelmente a saúde mental. Assim, o Conselho Federal de Enfermagem determinou à Comissão Nacional de Enfermagem em Saúde Mental para efetivar atendimento a esses profissionais que se encontram na linha de frente na atuação da pandemia.

Conclusão: para atingir esse objetivo, elaborou-se o projeto de Atendimento de Enfermagem em Saúde Mental aos profissionais de enfermagem na pandemia. Depois dos primeiros trinta dias de atendimentos, os sentimentos mais emergentes foram: ansiedade, medo, ambivalência, depressão e exaustão.

DESCRITORES: Enfermagem; Saúde Mental; Pandemias; Profissionais de Enfermagem.

COMO REFERENCIAR ESTE ARTIGO:

Humerez DC de, OhI RIB, Silva MCN da. Saúde mental dos profissionais de enfermagem do Brasil no contexto da pandemia Covid-19: ação do Conselho Federal de Enfermagem. Cogitare enferm. [Internet]. 2020 [acesso em "colocar data de acesso, dia, mês abreviado e ano"]; 25. Disponível em: http://dx.doi.org/10.5380/ ce.v25i0.74115.

${ }^{1}$ Enfermeira. Doutora em Enfermagem. Docente de Enfermagem da Universidade Federal de São Paulo. Coordenadora da Comissão Nacional de Enfermagem em Saúde Mental - COFEN. São Paulo, SP. Brasil. 9

${ }^{2}$ Enfermeira. Doutora em Enfermagem. Docente de Enfermagem da Universidade Federal de São Paulo. Membro da Comissão COFEN/MEC. São Paulo, SP, Brasil. $\bigcirc$

${ }^{3}$ Enfermeiro. Especialista em Enfermagem. Presidente do Conselho Federal de Enfermagem - COFEN. Brasília, DF, Brasil. 


\title{
MENTAL HEALTH OF BRAZILIAN NURSING PROFESSIONALS IN THE CONTEXT OF THE COVID-19 PANDEMIC: ACTION OF THE NURSING FEDERAL COUNCIL
}

\begin{abstract}
Objective: To reflect on the mental health of the Brazilian nursing professionals in the context of the COVID-19 pandemic.

Development: The current outbreak of the disease caused by the Coronavirus, COVID-19, is causing enormous damage to the entire world and tends to cause widespread panic in the population. Faced with this reality, the nursing professionals are part of one of the most affected groups, exposed to the risk of contagion and emotional pain that considerably affects mental health. Thus, the Federal Nursing Council appointed the Nursing National Commission on Mental Health to provide assistance to these professionals who are at the forefront of the pandemic.

Conclusion: In order to achieve this goal, the Nursing Care on Mental Health project for nursing professionals in the pandemic was elaborated. After the first thirty days of services, the most emerging feelings were anxiety, fear, ambivalence, depression, and exhaustion.
\end{abstract}

DESCRIPTORS: Nursing; Mental Health; Pandemics; Nursing Professionals.

\section{SALUD MENTAL DE LOS PROFESIONALES DE ENFERMERÍA DE BRASIL EN EL CONTEXTO DE LA PANDEMIA DE COVID-19: ACCION DEL CONSEJO FEDERAL DE ENFERMERİA}

\begin{abstract}
RESUMEN:
Objetivo: reflexionar sobre la salud mental de los profesionales de Enfermería de Brasil en el contexto de la pandemia de COVID-19.

Desarrollo: el brote actual de la enfermedad causada por el Coronavirus, COVID-19, está ocasionando enormes daños en todo el mundo y tiende a provocar pánico generalizado en la población. Frente a esta realidad, los profesionales de Enfermería forman parte de uno de los grupos más afectados, expuestos al riesgo de contagio y de padecer dolor emocional, que afecta considerablemente la salud mental. En consecuencia, el Consejo Federal de Enfermería designó a la Comisión Nacional de Enfermería en Salud Mental para que efectivice la atención de los profesionales que se encuentran en la primera línea de batalla contra la pandemia.

Conclusión: para lograr este objetivo, se elaboró el proyecto de Atención de Enfermería en Salud Mental para los profesionales de Enfermería en la pandemia. Después de los primeros treinta días de servicios, los sentimientos más expresados fueron los siguientes: ansiedad, miedo, ambivalencia, depresión y agotamiento.
\end{abstract}

DESCRIPTORES: Enfermería; Salud Mental; Pandemias; Profesionales de Enfermería. 
Conselho Federal de Enfermagem (COFEN) mantém atualizado os números dos profissionais de enfermagem ativos, sendo que o universo de profissionais registrados nos Conselhos Regionais de Enfermagem de todo país é de 2.283.808, distribuídos em 558.318 enfermeiros, 1.307.680 técnicos de enfermagem e 417.519 auxiliares de enfermagem ${ }^{(1)}$.

O trabalho é um processo no qual o ser humano, por meio das suas ações, controla e modifica a natureza, com a finalidade de produzir algo, e nesse mesmo processo, o ser humano modifica a si mesmo, pois imprime no trabalho as suas perspectivas de resultado ${ }^{(2)}$. $\mathrm{Na}$ saúde, o trabalho tem como finalidade a ação terapêutica da saúde. O objeto de trabalho da enfermagem é constituído por pessoas que necessitam de cuidados de saúde, com toda a complexidade e subjetividade do ser humano ${ }^{(2,3)}$.

O cuidado é para a enfermagem a essência de suas práticas e o aspecto predominante que a distingue das demais profissões na área da saúde, definida como arte, técnica, intuição e sensibilidade ${ }^{(4,5)}$. Cuidar de toda a complexidade humana constitui-se para o enfermeiro um desafio, pois suas demandas nunca cessam e nem poderão ser atendidas por completo. Durante o processo de adoecimento, quando surgem fragilidades, medos, ansiedades e desconfortos, a atenção à dimensão emocional do ser humano se faz mais necessária ainda ${ }^{(6)}$.

Esse manuscrito teve como objetivo refletir sobre a saúde mental dos profissionais de enfermagem do Brasil no contexto da pandemia COVID-19. Discorreu-se sobre cinco tópicos da temática: sofrimento emocional no cotidiano do trabalho da Enfermagem; pandemia COVID-19; sofrimento emocional dos profissionais de Enfermagem na pandemia COVID-19; o trabalho emocional do enfermeiro; e os sentimentos mais declarados pelos profissionais de Enfermagem.

\section{DESENVOLVIMENTO}

\section{Sofrimento emocional no cotidiano do trabalho da Enfermagem}

O exercício profissional da enfermagem é marcado por múltiplas exigências: lidar com dor, sofrimento, morte e perdas, a que se somam as condições desfavoráveis de trabalho e baixa remuneração. Esses fatores, em conjunto, propiciam a emergência de estresse, e até mesmo síndrome de burnout, termo criado para descrever o desgaste físico e psíquico de profissionais que lidam no exercício de suas funções, com altos níveis de envolvimento emocional. Tal situação se mantém em setores públicos e privados, justificando a realização de estudos que ressaltem, em seus resultados, a necessidade de se dar maior atenção à saúde dos profissionais de saúde ${ }^{(7-9)}$.

Mesmo a enfermagem tendo como objeto cuidar da vida, o resultado do seu trabalho, algumas vezes, pode resultar em danos irreversíveis que determinam sequelas graves e/ ou óbitos. Cuidar da vida em sofrimento e morte nos permite afirmar que o trabalho da enfermagem é gerador de sofrimento psíquico, sendo identificado como um trabalho penoso e insalubre para toda a equipe envolvida(7).

No cotidiano, o trabalho desenvolvido pela equipe de enfermagem é gerador de sentimentos ambíguos: ora colabora para vivências de prazer, ora para vivências de sofrimento. Isto se dá porque há possibilidade de ser útil enquanto servem, ajudam e confortam, porém, ao se deparar com o sofrimento alheio, a morte, a dor e/ou situações difíceis de ser superadas, o trabalhador sofre ${ }^{(10)}$.

Os profissionais são preparados para salvar vidas e grande parte do tempo se 
deparam com sofrimento e morte. É importante, então, que os profissionais de enfermagem entendam que o sofrimento e o prazer são sentimentos dialéticos no seu trabalho e essa compreensão é de grande relevância para a promoção da saúde dos trabalhadores e para a melhoria da qualidade da assistência prestada ${ }^{(11,12)}$.

\section{Pandemia COVID-19}

Estamos vivenciando na atualidade um momento de intenso desafio sob o ponto de vista de saúde, o atual surto da doença COVID-19 causada pelo Severe Acute Respiratory Syndrome Coronavirus 2(SARS-Cov2), o Coronavírus. Relatada pela primeira vez na província de Wuhan na China em 2019, a doença infecciosa COVID-19 se configura como uma nova enfermidade, diferente de outras causadas por Coronavírus, como a síndrome respiratória aguda grave (SARS) e a síndrome respiratória Oriente Médio (SROM). Trata-se de uma doença com rápida transmissibilidade entre indivíduos que podem ser sintomáticos ou não, cujos surtos podem crescer rápida e exponencialmente. Ainda não existem terapias ou vacinas que demonstrem tratar ou prevenir a COVID-19(13,14).

De acordo com os dados dos primeiros países afetados pela pandemia, cerca de $40 \%$ dos casos sofrerão uma doença leve, $40 \%$ experimentarão doenças moderadas, como pneumonia, $15 \%$ dos casos sofrerăo doença grave e $5 \%$ dos casos sofrerão doença grave, podendo levar à morte(14).

A pandemia da COVID-19 está ocasionando um prejuízo enorme para indivíduos, famílias, comunidades e sociedades em todo o mundo. A vida cotidiana mudou profundamente, a economia entrou em recessão e muitas das redes tradicionais de segurança social, econômica e de saúde pública nas quais as pessoas confiam em tempos difíceis foram submetidas a pressões sem precedentes ${ }^{(14-16)}$.

As pandemias tendem a provocar um pânico generalizado na população, principalmente quando o conhecimento sobre a doença ainda se encontra em construção. Há pesquisas em andamento que buscam medidas farmacológicas e vacinas para a COVID-19, mas até sua conclusão, a medida mais adotada mundialmente para o controle da transmissão é o isolamento social. Em isolamento, podem-se intensificar os sentimentos de desamparo, tédio, solidão, tristeza e reações comportamentais como alterações ou distúrbios de apetite, distúrbios do sono e conflitos interpessoais ${ }^{(16)}$.

Esses sentimentos e reações estão presentes no cotidiano da população e impactam o seu bem-estar. Temos também cerca de 3,5 milhões de trabalhadores do setor da saúde que enfrentam incertezas, anșeios e preocupações, afetando sua saúde mental e física neste período de pandemia. É nesse contexto que os profissionais da Enfermagem estão inseridos, atuando sem equipamentos de proteção adequados, no atendimento em geral ou em unidades de terapia intensiva, resgates, emergências, atenção primária, Unidade de Pronto Atendimento (UPAs) e enfermarias ${ }^{(17,18)}$.

\section{Sofrimento emocional dos profissionais de Enfermagem na pandemia COVID-19}

A Organização Mundial da Saúde (OMS) observa que os trabalhadores da enfermagem pressionados com essa situação apresentam altos níveis de ansiedade, acrescidos do risco de adoecer, provocando severos problemas de saúde mental e aumentando os casos da Síndrome de Burnout, além de gerar ansiedade, depressão e estresse associado(14).

O COFEN tem se manifestado em relação à falta dos equipamentos de proteção individual (EPIs), e destaca que a saúde do trabalhador ficará comprometida e o déficit de profissionais, ocasionado pelos afastamentos por conta da contaminação, poderá contribuir para o colapso do Sistema Único de Saúde ${ }^{(19)}$.

Segundo dados do COFEN, não existe um levantamento oficial do número de profissionais da saúde afastados em todo o Brasil. Porém, no mês de abril de 2020 foram 
realizadas fiscalizações in loco em 5.780 instituições de saúde no país. No levantamento situacional pelos Conselho Regionais de Enfermagem, identificou-se que 4.602 profissionais foram afastados por suspeita de COVID-19. Essa situação é grave, pois indica alto índice de contaminação na categoria, e insuficiência de EPls no país ${ }^{(20)}$.

Ainda segundo o COFEN, o número de enfermeiros e técnicos possivelmente infectados e afastados deu um salto na $2^{\mathrm{a}}$ quinzena do mês de abril. $\mathrm{O}$ aumento foi de $660 \%$, ou seja, de 158 foi para 1.203 casos. A maioria dos profissionais de enfermagem afastados tem entre 31 e 40 anos de idade, e $83 \%$ são mulheres ${ }^{(21)}$.

Diante desse processo cruel, em situação emergencial, o COFEN determinou à Comissão Nacional de Enfermagem em Saúde Mental para efetivar atendimento a ser realizado por enfermeiros especialistas, mestres ou doutores em Saúde Mental aos profissionais de enfermagem que se encontram na linha de frente na atuação da pandemia. E o processo se concretizou.

A base legal e ética para iniciar os atendimentos são a Lei $N^{\circ} 7498 / 86^{(22)}$, a Resolução COFEN N $N^{\circ} 599 / 2018^{(23)}$ e a Resolução COFEN N ${ }^{\circ} 564 / 2017^{(24)}$. A Lei $N^{\circ} 7498 / 86$ dispõe sobre a regulamentação do exercício da Enfermagem. A Resolução COFEN N ${ }^{\circ}$ 599/2018 estabelece parâmetros mínimos para assistência segura e humanizada na Enfermagem em Saúde Mental, sendo a base legal dos atendimentos. A Resolução COFEN N 564/2017 aprova o Código de Ética dos Profissionais de Enfermagem e explicita a base ética dos atendimentos ${ }^{(22-24)}$.

Ressalta-se que o sigilo e o anonimato dos profissionais atendidos estão sendo preservados. Foram cadastrados enfermeiros especialistas, mestres e ou doutores em Saúde Mental de todo o país, dada a emergência da situação. Feitos os convites a esses experts, aqueles que se disponibilizaram a atender via Live Chat aos profissionais de enfermagem (enfermeiros, técnicos de enfermagem e auxiliares de enfermagem inscritos no Sistema COFEN/Conselhos Regionais de Enfermagem) preencheram formulário específico com seus dados para cadastramento.

Para implementação do programa, tornou-se importante descrever, passo a passo, - objetivo, as ações previstas e os instrumentos necessários para alcançar os resultados esperados, bem como explicitar as etapas estabelecidas para sua realização.

Para o desenvolvimento desta iniciativa, houve a inserção de uma caixa de diálogo no site oficial do COFEN, localizada no canto inferior da tela, como motivação para o profissional entrar no Live Chat. O profissional que necessitar e desejar conversar com o enfermeiro de saúde mental deve clicar na caixa de diálogo, preencher dados como nome e inscrição profissional para ser encaminhado ao atendimento, que é realizado 24 horas por dia e nos sete dias da semana. A plataforma permite que até cinco enfermeiros realizem os atendimentos simultaneamente e há, no mínimo, um enfermeiro supervisor por turno disponível para apoiar os enfermeiros.

O potencial terapêutico do envolvimento interpessoal entre enfermeiros e profissional de enfermagem em sofrimento é um possível espaço de intervenção e escuta, uma vez que a enfermagem é a profissão cuja característica prioritária é a permanência junto ao paciente para o qual se desenvolve o cuidado. É preciso renunciar à fala, ao desejo de explicar, de convencer e de ser aquele que soluciona o sofrimento do outro.

Realizar apoio emocional implica ir além do óbvio, ser capar de detectar e reconhecer o subjetivo por trás das palavras e estar atento e sensível a cada gesto, olhar e expressão(25). A atenção às necessidades mais elevadas do ser humano requer escuta ativa e interações mais prolongadas, que permitam a formação de relacionamento terapêutico, vínculo e confiança. Assim, os cuidados de atenção à saúde são reajustados de forma que o indivíduo se torna centro do processo de cuidado, participando de seu planejamento e execução ${ }^{(26)}$.

\section{O trabalho emocional do enfermeiro}


Frente à situação emergencial, traçamos as diretrizes conceituais e concepções norteadoras para dar sustentação ao projeto de Atendimento de Enfermagem em Saúde Mental aos profissionais de enfermagem na pandemia COVID-19.

A ideia de intervenção implica em uma ação objetiva ou um fazer concreto em dada realidade, neste caso sendo atender o sofrimento emocional aos profissionais de enfermagem na pandemia COVID-19. Dada a situação emergencial, a metodologia definida para a intervenção respeitou a qualificação da equipe selecionada: enfermeiros especialistas, mestres ou doutores, que tivessem afinidade com o tema e condição técnica de organizar e intervir na execução das ações ${ }^{(24,26,27)}$.

A base utilizada é a corrente humanista, que ressalta aspectos positivos do ser humano, destacando que cada pessoa possui em si uma força de autorrealização que conduz ao desenvolvimento de uma personalidade mais saudável. A Escuta Empática permeia o processo de intervenção(28-30).

A teoria humanista de Maslow, usada na enfermagem como teoria das necessidades humanas básicas, apresenta ideias adotadas posteriormente por Carl Rogers na terapia centrada no cliente, assumindo assim um significado prático:

\begin{abstract}
"a pessoa possui possibilidades inimagináveis de se compreender, de modificar os conceitos que tem de si mesmo, suas posturas e seu comportamento e esse potencial pode ser liberado se a pessoa for conduzida a uma situação caracterizada por um clima favorável para o desenvolvimento psíquico"(31-33).
\end{abstract}

O pensamento rogeriano humanista aponta em direção à crença na orientação positiva do homem, a partir do conceito de tendência atualizadora. Faz-se necessário priorizar as condições facilitadoras que fazem emergir e impulsionar a operacionalização dessa tendência. Por meio de um clima de aceitação, autenticidade e empatia, a pessoa passa a implicar-se no processo de tornar-se pessoa, de ser o que é, para que a vida siga seu curso natural(33-35).

A noção de Tendência Atualizante é para Rogers o postulado fundamental da Abordagem Centrada na Pessoa, na medida em que conduz não só à satisfação das necessidades básicas, como também das mais complexas ${ }^{(33,34)}$. Nessa perspectiva, sempre que essa consonância não se verifica, a pessoa pode entrar numa espécie de incongruência consigo mesma, gerando uma desorganização entre a experiência real e a simbólica, o que leva a um comportamento desajustado e sofrimentos emocionais ${ }^{(34,35)}$.

A aceitação incondicional é o que garante o apreço das experiências do outro, ou o sofrimento emocional, independentemente de quaisquer condições, para que possibilite a compreensão mais realista de sua experiência - denominada compreensão empática ${ }^{(34)}$. Desse modo, ela se caracteriza como um modo de aceitar a pessoa tal como ela é, sem juízos de valor ou críticas. Assim, a consideração positiva incondicional é "uma aceitação calorosa de cada aspecto da experiência do cliente". Não há sentimentos que não possam ser expressos e "isto significa um cuidado com o cliente, mas não de forma possessiva. Implica numa forma de apreciar o cliente como uma pessoa individualizada"(33,34).

Saúde mental significa flexibilidade, opondo-se à rigidez. Ao fixar-se numa postura repetitiva e paralisadora, o profissional de enfermagem em sofrimento se vê impedido de exercer as múltiplas possibilidades de sua vida. Não negando os polos negativos da existência, tais como a resistência, inflexibilidade, mecanismos de defesa, incongruência e mal-estar, a concepção adotada enfatiza as condições positivas, facilitadoras de uma relação que delineia uma configuração mais plena, mais satisfatória, mais saudável(34).

\title{
Sentimentos mais declarados pelos profissionais de enfermagem
}

Durante os atendimentos, foram revelados os sentimentos mais declarados nos primeiros trinta dias de atendimentos, que foram organizados em: ansiedade, estresse, 
medo, ambivalência, depressão, exaustão.

- Ansiedade: falta de EPIs; pressão por parte da chefia imediata; com as notícias disponibilizadas pela mídia.

- Estresse: todo tempo chegando gente; morte como nunca houve.

- Medo do risco de se infectar; de infectar familiares.

- Ambivalência por parte da população (vizinhos, amigos) que os aplaudem, mas os discriminam, evitando contato.

- Depressão pela solidão, afastamento das famílias, morte dos companheiros de trabalho.

- Exaustão ou esgotamento emocional com o volume de trabalho.

\section{CONSIDERAÇÕES FINAIS}

mundo do trabalho se tornou, de forma rápida e surpreendente, um complexo monstruoso que gera muito sofrimento e sensação de esvaziamento, significando uma negatividade em sua existência, revelado nos depoimentos dos profissionais.

Uma estratégia para cuidar da saúde mental é o ouvir empático, planejado. Diante de uma pandemia como a que vivemos, os profissionais da enfermagem fazem parte de um dos grupos mais afetados, expostos ao risco de contágio e da dor emocional que afeta consideravelmente a saúde mental.

Uma boa proposta para todos é lembrar que a pandemia do novo Coronavírus não é o primeiro desafio enfrentado no planeta Terra e que não estamos sozinhos, mas fazemos parte de uma estratégia comunitária que pode salvar vidas.

\section{REFERÊNCIAS}

1. Conselho Federal de Enfermagem (COFEN). Enfermagem em números - Quantitativo de profissionais por regional. [Internet]. 2020 [acesso em 20 abr 2020]. Disponível em: http://www.cofen.gov.br/

enfermagem-em-numeros.

2. Forte ECN, Pires DEP de, Martins MMFP da S, Padilha MIC de S, Schneider DG, Trindade L de L. Processo de trabalho: fundamentação para compreender os erros de enfermagem. Rev. esc. enferm. USP [Internet]. 2019 [acesso em 28 abr 2020]; 53(e03489). Disponível em: https://doi.org/10.1590/s1980220x2018001803489.

3. Rodrigues MP, Melo RHV, Vilar RLA, Silva GSN, Silva AB. Reframing the work in health family strategy: challenges for the entire health care. Rev Bras Inov Tecnol Saúde [Internet]. 2017 [acesso em 28 abr 2020]; 7(2). Disponível em: https://doi.org/10.18816/r-bits.v7i2.6768.

4. Silva EKB, Silva Junior JNO da, Galindo Neto NM, Costa LS da, Rodrigues KF, Alexandre ACS. The art and science of caring: appreciation, established and outsiders in the autonomy of the nursing liberal professional. Rev. pesqui. cuid. fundam. [Internet]. 2019 [acesso em 28 abr 2020];11(2). Disponível em: http://ciberindex.com/c/ps/P112017.

5. Santos AG dos, Monteiro CF de S, Nunes BMVT, Benício CDAV, Nogueira LT. O cuidado em enfermagem analisado segundo a essência do cuidado de Martin Heidegger. Rev Cubana Enferm [Internet]. 2017 [acesso em 28 abr 2020]; 33(3). Disponível em: http://www.revenfermeria.sld.cu/index. 
6. Pinto AC, Garanhani ML, França TE de, Pierotti I. Conceito de ser humano nas teorias de enfermagem: aproximação com o ensino da condição humana. Pro-Posições [Internet]. 2017 [acesso em 28 abr 2020]; 28(supl.1). Disponível em: https://doi.org/10.1590/1980-6248-2015-0164.

7. Duarte M de LC, Glanzner CH, Pereira LP. O trabalho em emergência hospitalar: sofrimento e estratégias defensivas dos enfermeiros. Rev. Gaúcha Enferm [Internet]. 2018 [acesso em 28 abr 2020]; 39. Disponível em: https://doi.org/10.1590/1983-1447.2018.2017-0255.

8. Antunes M. Brazilian scientific production on psychic suffering and depression in nurses working in the emergency department. Rev Enferm Contemp [Internet]. 2017 [acesso em 28 abr 2020]; 6(1). Disponível em: http://dx.doi.org/10.17267/2317-3378rec.v6i1.1138.

9. Trapé TL, Campos RO. The mental health care model in Brazil: analyses of the funding, governance processes, and mechanisms of assessment. Rev. Saúde Pública [Internet]. 2017 [acesso em 28 abr 2020]; 51. Disponível em: https://doi.org/10.1590/s1518-8787.2017051006059.

10. Tostes MF do P, Silva AQ da, Garçon TL, Maran E, Teston EF. Duality between fulfilment and suffering in the work of the nursing staff in operating rooms. Rev. SOBECC. [Internet] 2017 [acesso em $28 \mathrm{abr}$ 2020]; 22(1). Disponível em: https://doi.org/10.5327/Z1414-4425201700010002.

11. Souza VS de, Silva DS da, Lima LV, Teston EF, Benedetti GM dos S, Costa MAR, et al. Qualidade de vida dos profissionais de enfermagem atuantes em setores críticos. Rev Cuid [Internet] 2018 [acesso em 28 abr 2020]; 9(2). Disponível em: http://dx.doi.org/10.15649/cuidarte.v9i2.506.

12. Rodrigues CCFM, Santos VEP, Sousa P. Patient safety and nursing: interface with stress and Burnout Syndrome. Rev. bras. enferm. [Internet]. 2017 [acesso em 28 abr 2020]; 70(5). Disponível em: https://doi. org/10.1590/0034-7167-2016-0194.

13. Viva Bem. Universo On-line (UOL). Coronavírus se espalha rápido, às vezes antes de infectados terem sintomas. [Internet]. 2020 [acesso em 15 abr 2020]. Disponível em: https://www.uol.com.br/vivabem/ noticias/redacao/2020/03/14/coronavirus-se-espalha-rapido-as-vezes-antes-de-infectados-teremsintomas.htm?cmpid=copiaecola.

14. World Heatlth Organization (WHO). Actualización de la estrategia frente a la COVID-19. Geneva: WHO; 2020 [acesso em 28 abr 2020]. Disponível em: https://www.who.int/docs/default-source/ coronaviruse/COVID-strategy-update-14april2020.pdf?sfvrsn=29da3ba0 19.

15. Lopez FG, Palotti PL de M, Barbosa SCT, Koga NM. Nota Técnica n. 30. Mapeamento dos profissionais de saúde no Brasil: alguns apontamentos em vista da crise sanitária da COVID-19. Instituto de Pesquisa Econômica Aplicada. [Internet]. 2020 [acesso em 15 abr 2020]. Disponível em: http:// repositorio.ipea.gov.br/bitstream/11058/9837/1/NT 30 Diest Mapeamento\%20dos\%20Profissionais\%20 de\%20Sa\%c3\%bade\%20no\%20Brasil.pdf.

16. UN News. COVID-19: mental health in the age of coronavirus. [Internet]. 2020 [acesso em $16 \mathrm{abr}$ 2020]. Disponível em: https://news.un.org/en/story/2020/03/1059542.

17. Weide JN, Vicentini ECC, Araújo MF de, Machado W de L, Enumo SRF. Cartilha para enfrentamento do estresse em tempos de pandemia. PUCRS/PUC-Campinas. [Internet]. 2020 [acesso em 17 abr 2020]. Disponível em: https://portal.coren-sp.gov.br/wp-content/uploads/2020/04/Cartilha-PsiCOVIDa.pdf.

18. Tavares V. A saúde dos que estão na linha de frente. Escola Politécnica de Saúde Joaquim Venâncio/ EPSJV/Fiocruz. [Internet]. 2020 [acesso em 17 abr 2020]. Disponível em: http://www.epsjv.fiocruz.br/ noticias/reportagem/a-saude-dos-que-estao-na-linha-de-frente.

19. Conselho Federal de Enfermagem (COFEN). Cancelamento de compra de EPIs preocupa o Cofen. [Internet]. 2020 [acesso em 17 abr 2020]. Disponível em: http://www.cofen.gov.br/cancelamento-decompra-de-epis-preocupa-o-cofen 78598.html.

20. Conselho Federal de Enfermagem (COFEN). Fiscalização identifica 4.602 profissionais afastados por 
suspeita de COVID-19. [Internet]. 2020 [acesso em 28 abr 2020]. Disponível em: http://www.cofen.gov.br/ fiscalizacao-identifica-4-602-profissionais-afastados-por-suspeita-de-COVID-19 79347.html.

21. Conselho Federal de Enfermagem (COFEN). COVID-19 faz vítimas entre profissionais da saúde no Brasil. [Internet]. 2020 [acesso em 28 abr 2020]. Disponível em: http://www.cofen.gov.br/COVID-19-fazvitimas-entre-profissionais-da-saude-no-brasil 78979.html.

22. Conselho Federal de Enfermagem. Lei n. 7498/86 de 25 de junho de 1986. Dispõe sobre a regulamentação do exercício da Enfermagem e dá outras providências. Brasília: COFEN; 1986 [acesso em 16 abr 2020]. Disponível em: http://www. cofen.gov.br/lei-n-749886-de-25-de-junho-de-1986 4161.html.

23. Conselho Federal de Enfermagem. Resolução n. 599/2018. Dispõe sobre o estabelecimento de parâmetros mínimos para assistência segura e humanizada na Enfermagem em Saúde Mental. Brasília: COFEN; 2018 [acesso em 16 abr 2020]. Disponível em: http://www.cofen.gov.br/resolucao-cofenno-599-2018 67820.html.

24. Conselho Federal de Enfermagem. Resolução n. 564/2017. Aprova o Código de ética dos profissionais de Enfermagem. Brasília: COFEN; 2017 [acesso em 16 abr 2020]. Disponível em: http://www. cofen.gov.br/resolucao-cofen-no-5642017 59145.html.

25. Monteiro P de V, Almeida ANS de, Pereira MLD, Freitas MC de, Guedes MVC, Silva L de F da. When body care is not enough: the emotional dimension of nursing care. REME - Rev Min Enferm. [Internet]. 2016 [acesso em 28 abr 2020]; 20(e957). Disponível em: http://www.dx.doi.org/10.5935/14152762.20160026.

26. World Health Organization (WHO). Humanitarian Intervention Guide (mhGAP-HIG): clinical management of mental, neurological and substance use conditions in humanitarian emergencies. [Internet]. 2015 [acesso em 16 abr 2020]. Disponível em: https://www.who.int/mental health/publications/ mhgap hig/en/.

27. Lemos PM, Cavalcante Júnior FS. Psicologia de orientação positiva: uma proposta de intervenção no trabalho com grupos em saúde mental. Ciênc. Saude Colet [Internet]. 2009 [acesso em 16 abr 2020; 14(1). Disponível em: https://doi.org/10.1590/S1413-81232009000100029.

28. Silva JAM da, Siegmund G, Bredemeier J. Intervenções em nos atendimentos psicológicos online. Trends Psychiatry Psychother. [Internet]. 2015 [acesso em 16 abr 2020]; 37(4). Disponível em: https://doi. org/10.1590/2237-6089-2014-0026.

29. Organização Pan-Americana de Saúde - Brasil (OPAS/BR). Manejo Clínico de Condições Mentais, Neurológicas e por Uso de Substâncias em Emergências Humanitárias. Guia de Intervenção Humanitária mhGAP (GIH-mhGAP). Brasília: Organização Pan-Americana da Saúde; 2020 [acesso em 16 abr 2020]. Disponível em: https://iris.paho.org/handle/10665.2/51948.

30. Maslow A. Motivação e Personalidade. 2. ed. [Internet] Massachussets: Harper \& Row Editores; 2018. [acesso em $17 \mathrm{abr}$ 2020]. Disponível em: https://docero.com.br/doc/s81s0s.

31. Ferreira A, Demutti CM, Gimenez PEO. A Teoria das Necessidades de Maslow: a influência do nível educacional sobre a sua percepção no ambiente de trabalho. In: 13 SEMEAD - Seminários em Administração; 2010 set. [Internet]. 2010 [acesso em 16 abr 2020]. Disponível em: https://www.etica.eco. br/sites/textos/teoria-de-maslow.pdf.

32. Branco PCC, Cirino S. Reception and circulation of carl Rogers's Humanistic Psychology in Brazil. Rev. psicol [Internet]. 2017 [acesso em 17 abr 2020]; 26(2). Disponível em: https://scielo.conicyt.cl/scielo. php?script=sci abstract\&pid=S0719-05812017000200106\&lng=en\&nrm=iso.

33. Rogers CR. Tornar-se Pessoa. 6. ed. [Internet]. São Paulo: WMF; 2017 [acesso em 16 abr 2020]. Disponível em: https://docero.com.br/ doc/n8nsc0.

34. Miranda ABS de. A abordagem centrada na pessoa. Psicologado [Internet]. 2013 [acesso em 16 abr 2020]; Disponível em: https://psicologado.com.br/abordagens/centrada-na-pessoa/a-abordagemcentrada-na-pessoa-acp. 
35. Rogers CR. Um jeito de ser. São Paulo: EPU; 1996.

Recebido: 04/05/2020

Finalizado: 15/05/2020

Editora associada: Luciana Puchalski Kalinke

Autor Correspondente:

Dorisdaia Carvalho de Humerez

Universidade Federal de São Paulo

R. Sena Madureira, 1500 - 04021-001 - São Paulo, SP, Brasil

E-mail: doris_daia@yahoo.com.br

Contribuição dos autores:

Contribuições substanciais para a concepção ou desenho do estudo; ou a aquisição, análise ou interpretação de dados do estudo - DCH, RIBO, MCNS

Elaboração e revisão crítica do conteúdo intelectual do estudo - DCH, RIBO, MCNS

Aprovação da versão final do estudo a ser publicado - DCH, RIBO, MCNS

Responsável por todos os aspectos do estudo, assegurando as questões de precisão ou integridade de qualquer parte do estudo - DCH, RIBO, MCNS 